\title{
Do Concentration or Activity of Selenoproteins Change in Acute Stroke Patients? A Systematic Review and Meta-Analyses
}

\author{
Sabrina Tamburrano ${ }^{a, b}$ Sarah Rhodes ${ }^{c}$ Ioana-Emilia Mosneaga, d \\ Lucy Roberts $^{a}$ d Madeleine E.D. Hurry ${ }^{a}$ d, e John R. Grainger ${ }^{b}$ e \\ Tovah N. Shaw ${ }^{f}$ Craig J. Smitha, e, Stuart M. Allan ${ }^{a, d, e}$
}

a Geoffrey Jefferson Brain Research Centre, Faculty of Biology, Medicine and Health, Manchester Academic Health Science Centre, University of Manchester, Manchester, UK; ${ }^{b}$ Division of Infection, Immunity and Respiratory Medicine, School of Biological Sciences, Faculty of Biology, Medicine and Health, University of Manchester, Manchester, UK; ${ }^{c}$ Centre for Biostatistics, Division of Population Health, Health Services Research \& Primary Care, School of Health Sciences, Manchester Academic Health Science Centre, University of Manchester, Manchester, UK; ${ }^{d}$ Division of Neuroscience and Experimental Psychology, School of Biological Sciences, Faculty of Biology, Medicine and Health, University of Manchester, Manchester, UK; éLydia Becker Institute of Immunology and Inflammation, Faculty of Biology, Medicine and Health, Manchester Academic Health Science Centre, University of Manchester, Manchester, UK; fInstitute of Immunology and Infection Research, School of Biological Sciences, University of Edinburgh, Ashworth Laboratories, Edinburgh, UK; ${ }^{9}$ Manchester Centre for Clinical Neurosciences, Salford Royal NHS Foundation Trust, Salford, UK

\section{Keywords}

Glutathione peroxidase · Haemolysate · Plasma ·

Selenoprotein · Serum · Stroke

\begin{abstract}
Introduction: Stroke is characterized by deleterious oxidative stress. Selenoprotein enzymes are essential endogenous antioxidants, and detailed insight into their role after stroke could define new therapeutic treatments. This systematic review aimed to elucidate how blood selenoprotein concentration and activity change in the acute phase of stroke. Methods: We searched PubMed, EMBASE, and Medline databases for studies measuring serial blood selenoprotein concentration or activity in acute stroke patients or in stroke patients compared to non-stroke controls. Metaanalyses of studies stratified by the type of stroke, blood
\end{abstract}

compartment, and type of selenoprotein measurement were conducted. Results: Eighteen studies and data from 941 stroke patients and 708 non-stroke controls were included in this review. Glutathione peroxidase (GPx) was the only identified selenoprotein, and its activity was most frequently measured. Results from 12 studies and 693 patients showed that compared to non-stroke controls in acute ischaemic stroke patients, the GPx activity increased in haemolysate (standardized mean difference [SMD]: 0.27, 95\% Cl: 0.07-0.47) but decreased in plasma (mean difference [MD]: $-1.08 \mathrm{U} / \mathrm{L}, 95 \% \mathrm{Cl}:-1.94$ to -0.22 ) and serum (SMD: $-0.54,95 \% \mathrm{Cl}:-0.91$ to -0.17$)$. From 4 identified studies in 106 acute haemorrhagic stroke patients, the GPx activity decreased in haemolysate (SMD: $-0.40,95 \% \mathrm{Cl}$ : -0.68 to -0.13 )

John R. Grainger, Tovah N. Shaw, Craig J. Smith, and Stuart M. Allan contributed equally. karger@karger.com www.karger.com/ced

Karger $\stackrel{\text { ' }}{5}$

BOPEN ACCESS
(C) 2022 The Author(s)

Published by S. Karger AG, Basel

This article is licensed under the Creative Commons Attribution 4.0 International License (CC BY) (http://www.karger.com/Services/ OpenAccessLicense). Usage, derivative works and distribution are permitted provided that proper credit is given to the author and the original publisher.
Correspondence to:

Sabrina Tamburrano, sabrina.tamburrano@ postgrad.manchester.ac.uk Craig J. Smith, craig.smith-2@ manchester.ac.uk 
and remained unchanged in plasma (MD: $-0.10 \mathrm{U} / \mathrm{L}, 95 \% \mathrm{Cl}$ : -0.81 to 0.61 ) and serum (MD: $-5.00 \mathrm{U} / \mathrm{mL}, 95 \% \mathrm{Cl}:-36.17$ to 26.17) compared to non-stroke controls. Results from studies assessing the GPx activity in the haemolysate compartment were inconsistent and characterized by high heterogeneity. Conclusions: Our results suggest a reduction of the blood GPx activity in acute ischaemic stroke patients, a lack of evidence regarding a role for GPx in haemorrhagic stroke patients, and insufficient evidence for other selenoproteins.

(c) 2022 The Author(s)

Published by S. Karger AG, Basel

\section{Introduction}

The use of antioxidant therapies is a promising direction for the development of new treatments for stroke. Oxidative stress is a common feature of ischaemic and haemorrhagic stroke [1], and several preclinical studies [2-5] have demonstrated that an increase in antioxidant enzymes could be protective against stroke. Since oxidative stress is a key factor in the pathophysiology of stroke and could be used to develop novel therapies, further understanding of endogenous antioxidant mechanisms occurring after stroke is crucial.

Some selenium-dependent proteins, or selenoproteins, are key elements in the endogenous antioxidant system. One of the most important selenoprotein enzymes included in the first line of antioxidant defence is glutathione peroxidase $(\mathrm{GPx})$, which functions to neutralize hydrogen peroxide $\left(\mathrm{H}_{2} \mathrm{O}_{2}\right)$ generated by the dismutation of superoxide anions [6]. $\mathrm{H}_{2} \mathrm{O}_{2}$ can easily cross cell membranes, leading to wide-spread oxidative stress-induced damage during stroke [7]. As an $\mathrm{H}_{2} \mathrm{O}_{2}$ scavenger, GPx may be able to interrupt this detrimental oxidative stress propagation in the brain. In agreement with a potential protective role for GPx in stroke, polymorphisms causing a reduction in its transcriptional activity are associated with increased risk of ischaemic stroke and thrombosis [8-10]. Furthermore, in addition to increasing the transcription of several selenoprotein genes in the brain, intraperitoneal injection of selenium-containing peptides in a mouse model of intracerebral haemorrhage (ICH) results in the reduction of oxidative stress, excitotoxicity, and consequently haematoma volume [3]. One of the most promising antioxidant clinical trials in stroke concerned the use of ebselen, a seleno-organic compound with mimetic antioxidant activity to GPx. Oral administration of ebselen improved neurological deficits and reduced ischaemic volume in rodent models of focal cerebral ischaemia but, in clinical trials, showed beneficial effects only until 1 month post-stroke $[5,11]$.

To date, the precise role of selenoproteins in the aetiology and pathophysiology of stroke is still controversial. In this study, we aimed to clarify how selenoprotein concentration and activity change in the acute phase of stroke by undertaking a systematic review and meta-analysis of observational clinical studies.

\section{Material and Methods}

This systematic review was conducted in accordance with the Preferred Reporting Items for Systematic Review and Meta-Analysis (PRISMA) guidelines, and the protocol was preregistered in the PROSPERO database (CRD42020193646).

\section{Search Strategy and Selection Criteria}

The search of PubMed and OVID (EMBASE and Medline) was conducted up to 7th December 2020. References were also searched for relevant articles and reviews, with no additional studies found.

Four authors (S.T., I.-E.M., L.R., and M.E.D.H.) independently screened articles for inclusion based on title, abstract, and full text. Observational studies were eligible if they measured selenoprotein concentration and/or activity in blood compartments (haemolysate, serum, plasma, or whole blood) in people aged 18 years or over, with a diagnosis of ischaemic or haemorrhagic stroke (for keyword search strategy see online suppl. section S1; see www.karger.com/doi/10.1159/000520856 for all online suppl. material). Based on the aims of this study, articles were further considered based on comparison between at least one of the following: selenoprotein concentration and/or activity in stroke patients sampled within 3 days of hospital admission and non-stroke control, and selenoprotein concentration and/or activity in stroke patients sampled within 3 days of hospital admission and the same patients from 7 days to 6 months after stroke onset (Fig. 1; for detailed inclusion/exclusion criteria, see online suppl. section S2).

\section{Data Extraction and Quality Assessment}

Three authors (S.T., I.-E.M., and L.R.) independently performed the data extraction, and all discrepancies were resolved by discussion. The quality of the articles was assessed using a modified 14-point National Heart, Lung, and Blood Institute (NIHLB) questionnaire for observational and cross-sectional studies (online suppl. data S3). Based on the number of positive answers, 4 authors (S.T., I.-E.M., L.R., and M.E.D.H.) independently and arbitrarily assigned the range boundaries of "poor," "fair," and "good" as a rating of quality. This range was discussed and agreed between the review team. A number of positive answers $\leq 4$ define a "poor" rating, between 5 and 9 define a "fair" rating, $\geq 10$ define a "good" rating.

\section{Statistical Analysis}

All statistical analyses were performed using Review Manager version 5.3 software. The main characteristics of all included articles were collected for narrative synthesis and are shown in Table 1 and online Supplementary Table S4. For the meta- 


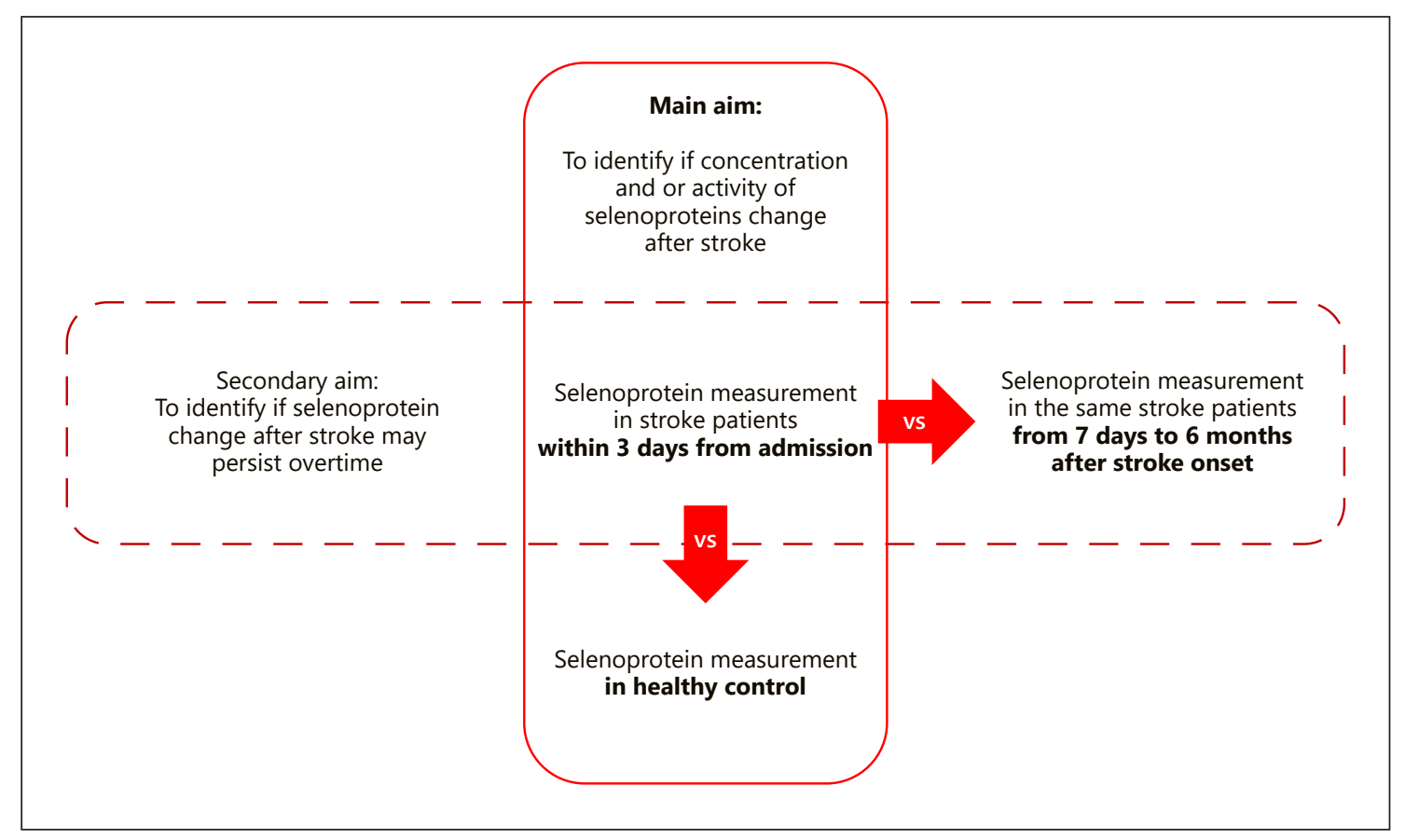

Fig. 1. Study scheme illustrating the 2 aims of the systematic review.

Table 1. Overview of the included study characteristics [12-29]

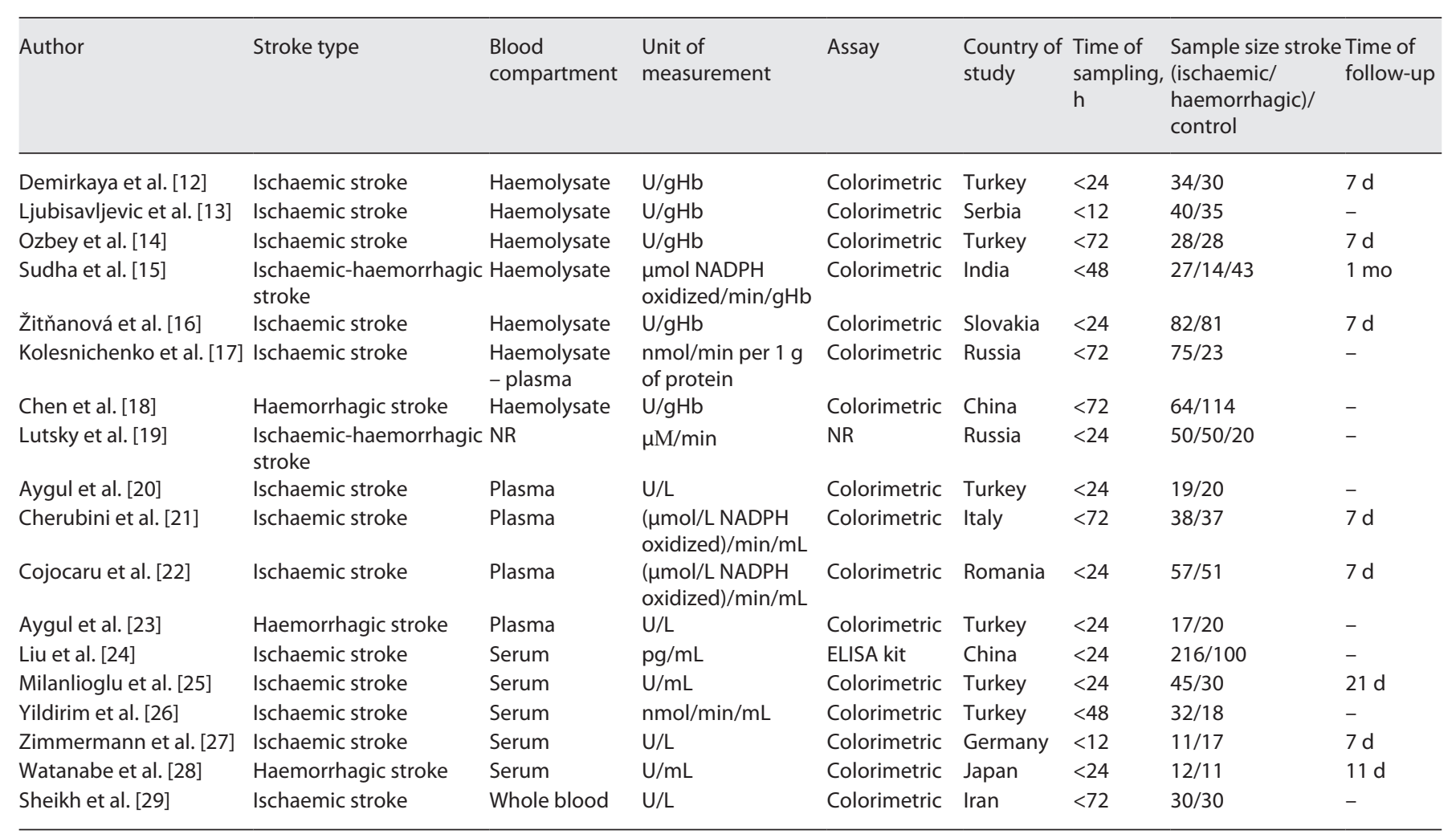

Studies sorted by the blood compartment and type of stroke. 


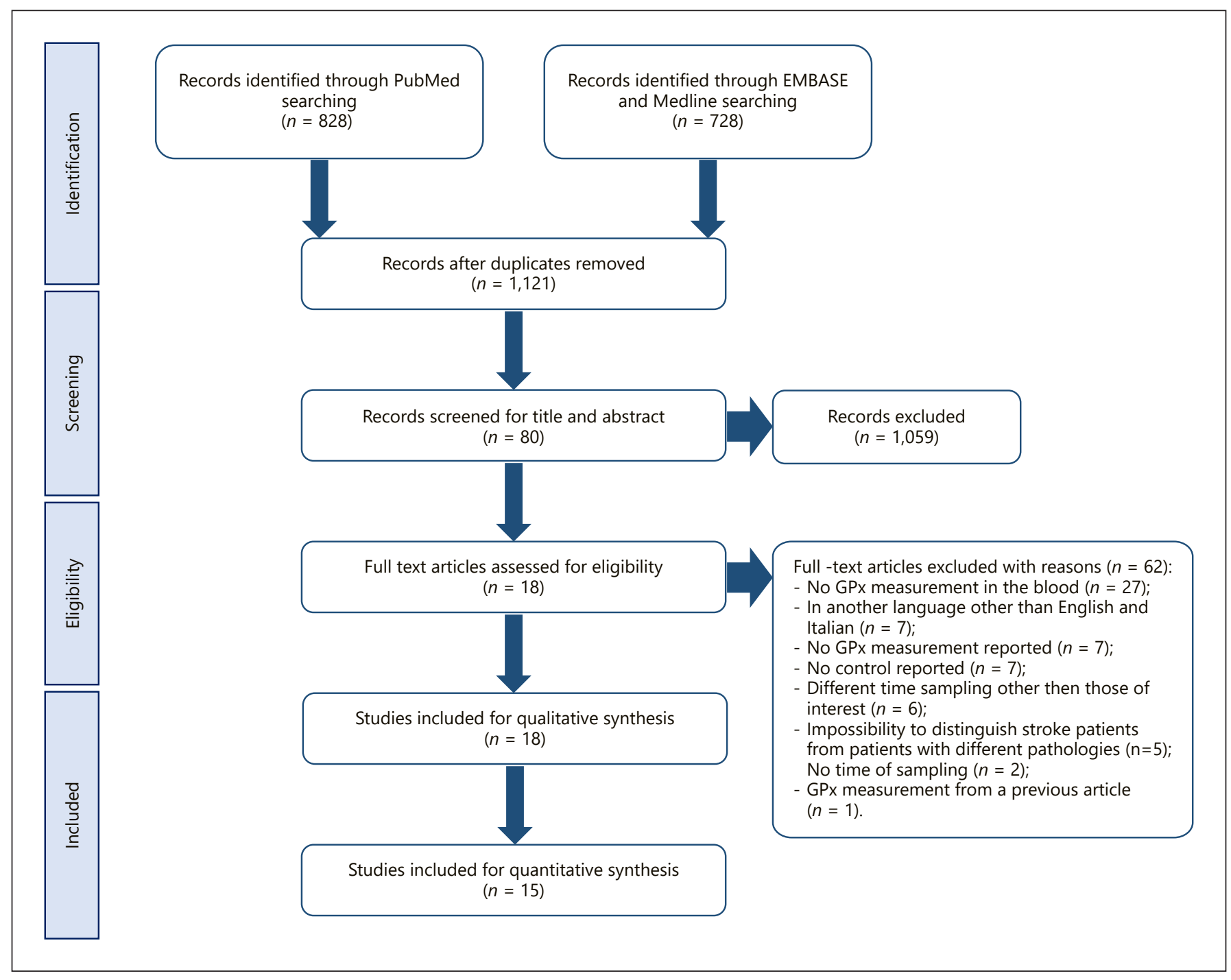

Fig. 2. PRISMA flowchart showing search strategy and the number of included/excluded studies for the systematic review and meta-analysis. GPx, glutathione peroxidase; PRISMA, Preferred Reporting Items for Systematic Review and Meta-Analysis.

analysis, studies were synthesized considering the GPx activity or concentration measurement in the blood. Sample size per group, mean of the blood GPx activity or concentration, and their standard deviation (SD), standard error, or interquartile range were extracted. When possible, studies measuring the GPx activity or concentration in the same compartment were standardized for unit of measurement. Depending on the blood compartment, the following units of measurement were used: U/ $\mathrm{gHb}$ for haemolysate GPx activity, U/L for plasma GPx activity, $\mathrm{U} / \mathrm{L}$ for serum GPx activity, pg/mL for serum GPx concentration, and U/L for whole blood GPx activity. When raw values were shown in a graph only, the online graphical tool WebPlotDigitizer (https://automeris.io/WebPlotDigitizer/) was used to extract data from figures. Where interquartile range was report- ed, SD was measured using the method reported in the study by Wan et al. [30]. When GPx measurement was stratified in patients based on stroke severity or outcome, the groups were combined using a weighted mean.

For the secondary analysis, a crossover design was preferred. SD and standard error were estimated and used for a generic inverse-variance outcome analysis. An additional analysis was conducted investigating a possible correlation with National Institutes of Health Stroke Scale (NIHSS) severity and modified Rankin Scale (mRS) outcome. For this purpose, data from studies showing the mean GPx activity in patients stratified for the NIHSS or mRS were also extracted.

Heterogeneity among studies was assessed by Cochrane $Q$ and $I^{2}$ statistics. Based on heterogeneity and variance between studies, 


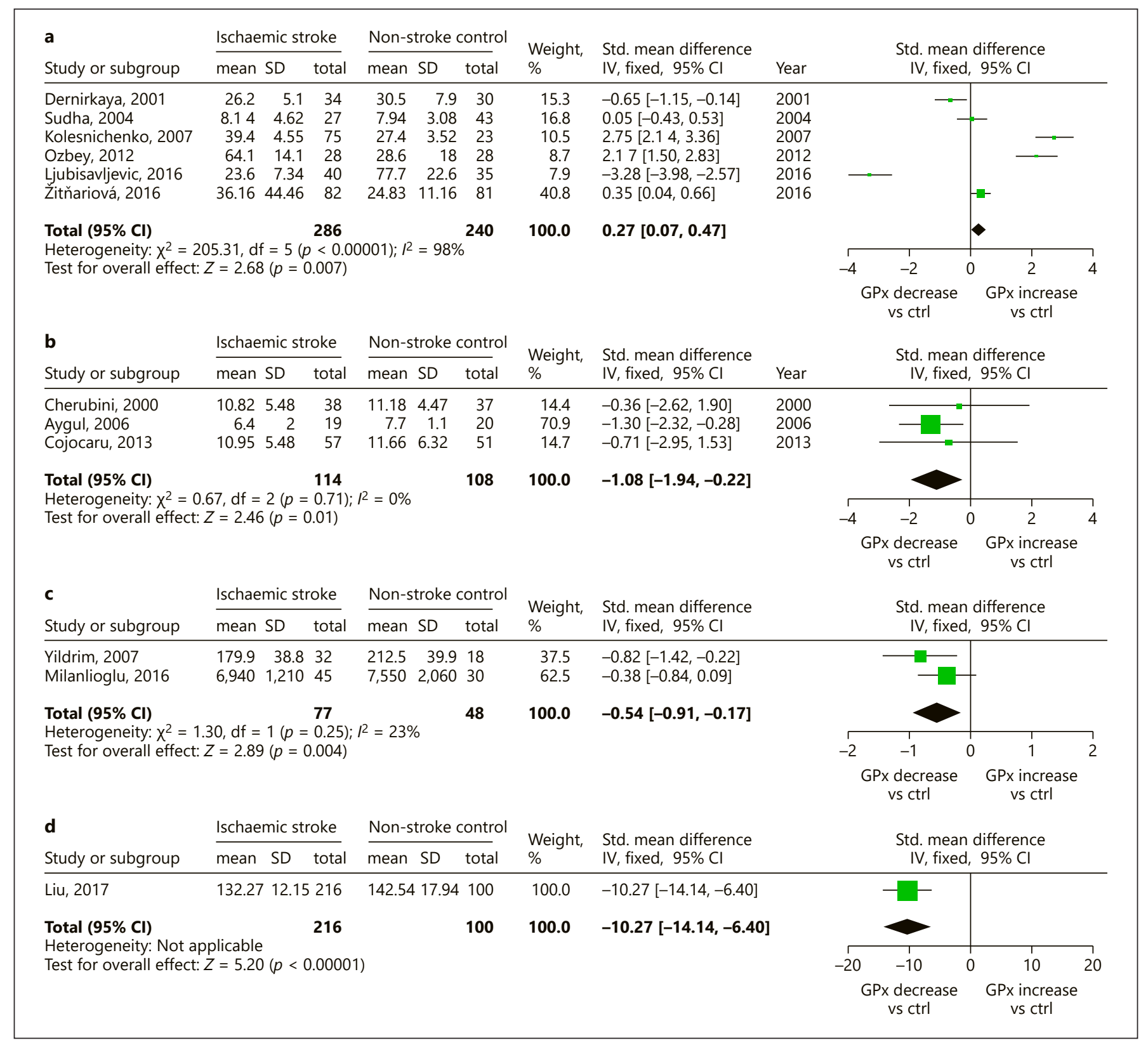

Fig. 3. Comparison of GPx activity in haemolysate (a), plasma (b), and serum (c), and GPx concentration in serum (d) of ischaemic stroke patients and non-stroke controls. Forest plot of fixed effect of SMD (a, c) or MD (b, d) and 95\% CI. GPx, glutathione peroxidase; MD, mean difference; SMD, standardized mean difference.

mean difference (MD) or standardized mean difference (SMD) was preferred. When SMD was applied, not normalized units of measurement were adopted. Considering the similarity of the studies in terms of design, patients recruited, and outcome measured, including time of sampling and compartment analyzed, fixed-effect meta-analysis has been preferred.

Circulating Selenoprotein Status in Stroke Patients

\section{Results}

Descriptive Analysis

The initial PubMed search identified 828 studies, and an additional search on OVID (EMBASE and Medline) identified 728 studies. After duplicate removal, 1,121 studies were identified and screened for the inclusion/ex- 


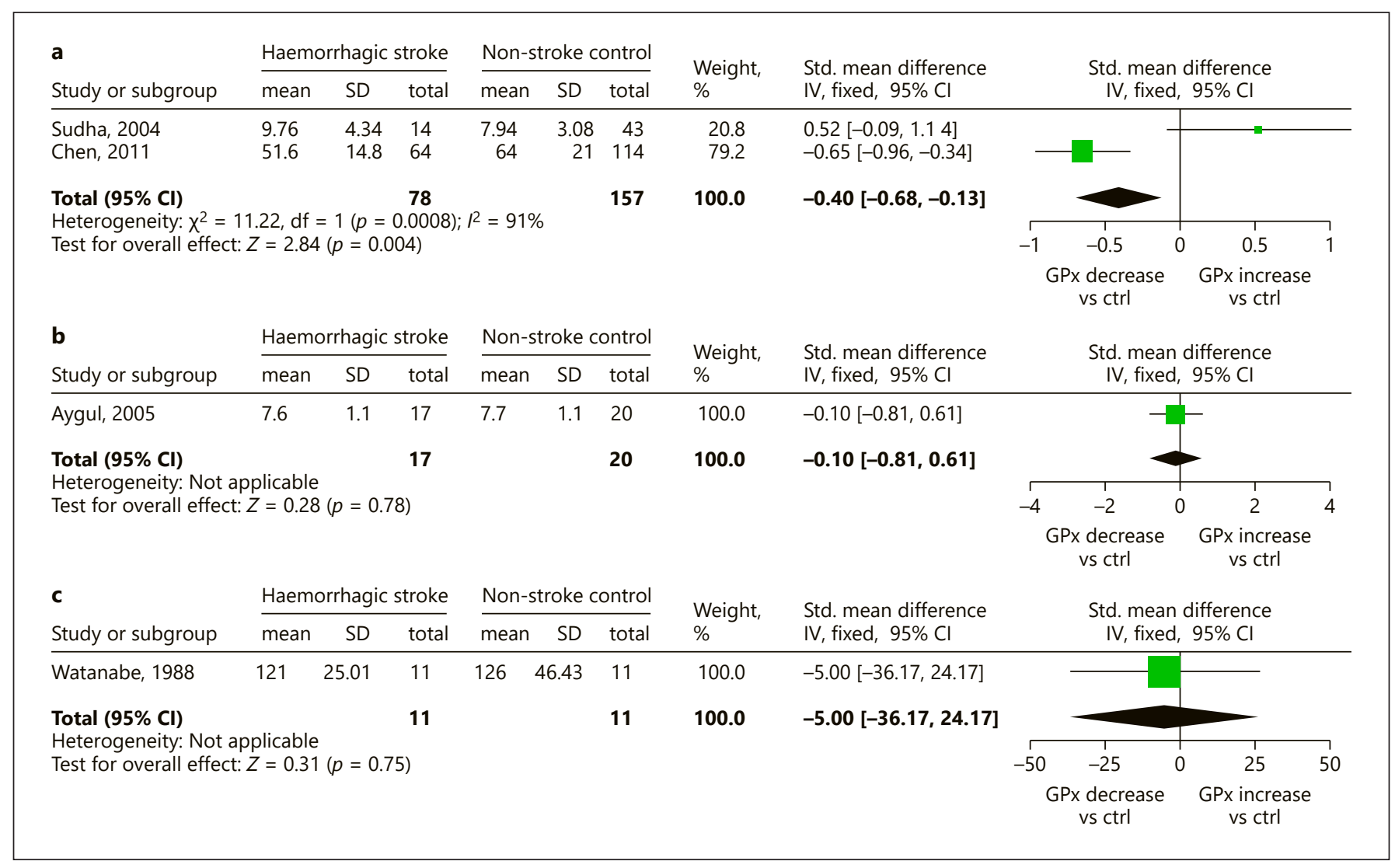

Fig. 4. Comparison of GPx activity in haemolysate (a), plasma (b), and serum (c) of haemorrhagic stroke patients and non-stroke control. Forest plot of fixed effect of SMD (a) or MD (b, c) and 95\% CI. GPx, glutathione peroxidase; MD, mean difference; SMD, standardized mean difference.

clusion criteria. All included studies measured the activity/concentration of the same selenoprotein, GPx. Eighteen studies were eligible for qualitative analysis, 16 of which were also eligible for quantitative analysis (Fig. 2).

Overall, data from 941 stroke patients were included in this review, with a mean age of $\sim 65$ years, with all studies conducted in Asia and Europe. Most of the clinical patient and control characteristics were not reported. Only a few studies reported the frequency of excess alcohol consumption $(n=3)$, smoking status $(n=8)$, hypertension $(n=9)$, and diabetes in the stroke patients $(n=7)$. Only 1 study recruited patients with a previous history of stroke, whereas in most of the studies, this information was not reported. As a possible confounding factor, we sought dietary information, but no studies reported these data, and instead, 4 studies excluded stroke patients who had been using antioxidants during the months preceding the enrolment (see online suppl. Table S4).
Most of the studies $(n=15)$ investigated GPx change in ischemic stroke patients, whereas only 5 investigated GPx change in haemorrhagic stroke patients. GPx activity was the measurement most commonly analyzed ( $n=$ 17). Only 1 study analyzed GPx concentration in serum.

In order to minimize variance and heterogeneity of the studies, they were stratified by the type of stroke, blood compartments, and GPx concentration/activity (see online suppl. section S6). Since 2 studies either did not report the blood compartment from which the analysis was performed [19] or included $7 / 11$ patients with minor or no permanent deficit in the ischaemic stroke group [27], they were only eligible for the qualitative analysis.

The quality rate of most included articles was fair (67\%), and the most frequent value was 6 (5-7 points; online suppl. Table S5). Lower scores were mostly due to the lack of information regarding the participation rate of eligible persons, sample size justification, if outcome assessors were blinded to the exposure status of participants, and if con- 


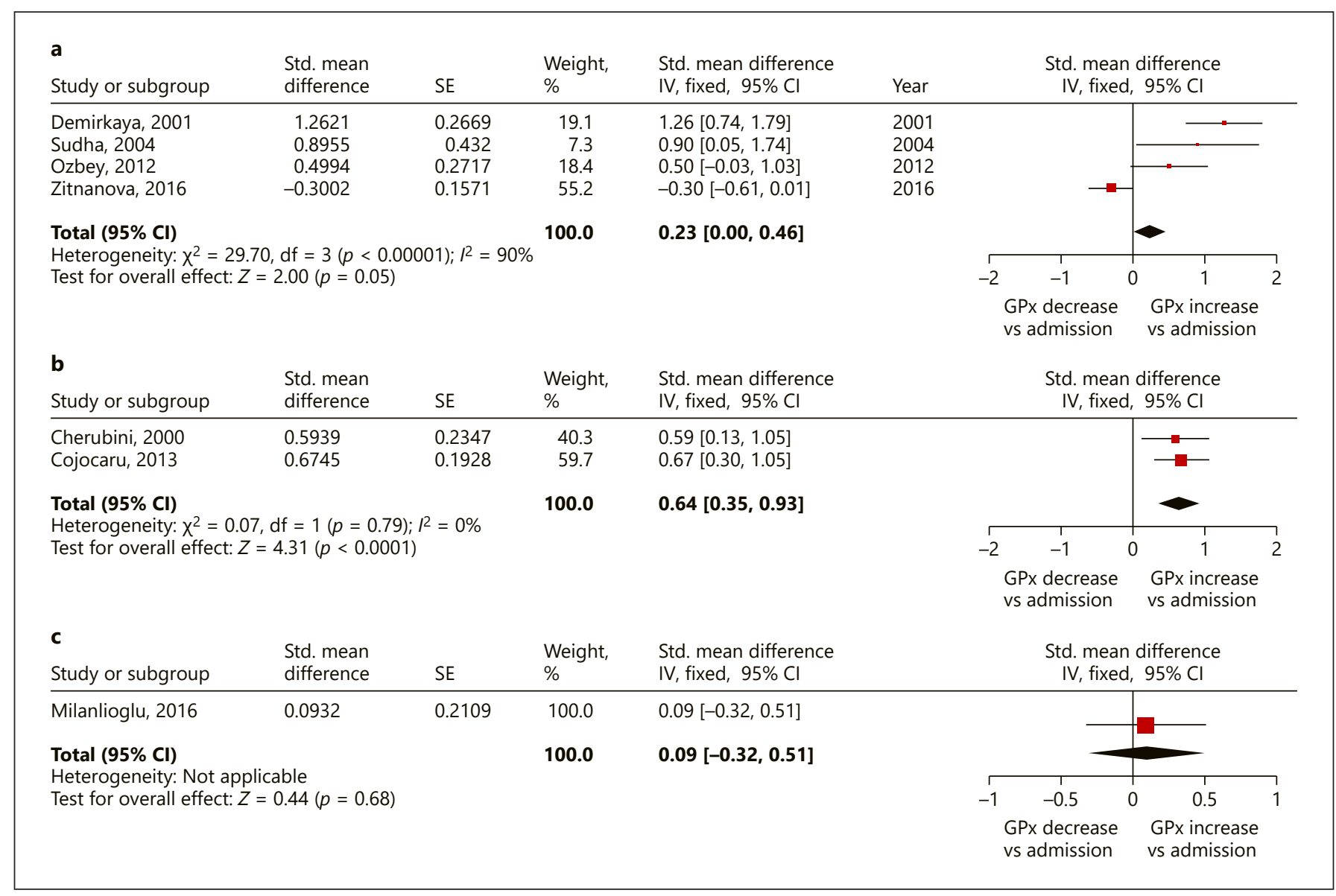

Fig. 5. Comparison of GPx activity in the haemolysate (a), plasma (b), and serum (c) of ischaemic stroke patients between 7 days and 1 month from stroke onset and the same patients at admission. Forest plots of fixed effect of SMD and 95\% CI. GPx, glutathione peroxidase; SMD, standardized mean difference.

founding variables were measured and adjusted statistically for their impact. Given the small number of studies, it was not possible to express publication bias as a funnel plot.

\section{Quantitative Synthesis: Meta-Analyses \\ Main Objective}

In haemolysate, the GPx activity was increased in ischaemic stroke patients compared to non-stroke controls (SMD: 0.27 , 95\% CI: 0.07-0.47, Fig. 3a). In contrast, the GPx activity was decreased in haemorrhagic stroke patients compared to non-stroke controls (SMD: -0.40 , $95 \%$ CI: -0.68 to -0.13 , Fig. $4 a$ ). In both analyses, despite the studies being grouped to minimize the interstudy heterogeneity, $I^{2}$ was found to be $>90 \%$, indicating substantial heterogeneity. This suggests a serious inconsistency between the studies, with SMD ranging between -3.28 and 2.75 in ischaemic stroke analysis, and -0.65 and 0.52 in haemorrhagic stroke analysis. To understand whether study quality could affect the heterogeneity, analysis of studies grouped for the quality rate has been performed. Two ischaemic stroke studies and 1 haemorrhagic stroke study were rated as "poor" and showed a higher effect size than the studies rated as "good/fair" (see online suppl. data S7 and S8). These data might suggest a relationship between study quality and effect size.

In plasma, the GPx activity was significantly reduced in ischaemic stroke patients compared to non-stroke controls (MD: $-1.08 \mathrm{U} / \mathrm{L}, 95 \% \mathrm{CI}:-1.94$ to -0.22 , Fig. $3 \mathrm{~b}$ ). One study [17] used different units of measurements to the others, and the post hoc decision was made to exclude it from the meta-analysis. In the same compartment, the GPx activity remained unchanged in haemorrhagic stroke patients compared to non-stroke controls (MD: -0.10 U/L, 95\% CI: -0.81 to 0.61 , Fig. 4 b). 
In serum, the GPx activity and concentration were significantly reduced in ischaemic stroke patients compared to non-stroke controls (SMD: $-0.54,95 \% \mathrm{CI}:-0.91$ to -0.17 ; MD: $-10.27 \mathrm{pg} / \mathrm{mL}, 95 \% \mathrm{CI}:-14.14$ to -6.40 , respectively, Fig. 3c, d). In the same compartment, the GPx activity remained unchanged in haemorrhagic stroke patients compared to non-stroke controls (MD: $-5.00 \mathrm{U} /$ mL, 95\% CI: -36.17 to 26.17 , Fig. $4 c$ ).

The whole blood compartment was investigated in only 1 study, which reported a comparison between ischaemic stroke patients and non-stroke controls. From this, the GPx activity was significantly reduced in ischemic stroke patients compared to non-stroke controls (SMD: $-0.58,95 \%$ CI: -1.10 to -0.06 , online suppl. Fig. S9).

According to the results from plasma, serum, and whole blood, the study by Lutsky et al. [19], which did not report in which blood compartment the analyses were performed, showed a significant decrease of the GPx activity in ischaemic stroke patients compared to nonstroke controls (SMD: $-4.81,95 \% \mathrm{CI}:-5.77$ to -3.84 , online suppl. Fig. S10a). However, the haemorrhagic stroke analysis in this study was not consistent with the other 4 included studies. Whereas a possible decrease of the GPx activity in haemolysate and no changes in plasma and serum are reported (Fig. 4), Lutsky et al. [19] described a significant increase of the GPx activity in haemorrhagic stroke patients compared to non-stroke controls (SMD: 39.88, 95\% CI: 33.06-46.70, online suppl. Fig. S10b).

\section{Secondary Objective}

For the secondary objective, 8 studies included followup analysis: 7 measured the activity of GPx in ischaemic stroke patients, whereas only 1 study included haemorrhagic stroke patients. In haemolysate of ischaemic stroke patients, the GPx activity increased between 7 days and 1 month from stroke onset, compared to the value of the same patients on admission (SMD: 0.23 , 95\% CI: $0.00-$ 0.46 , Fig. 5a). Although subgroup analysis of the study quality rate showed that the poor quality rated study had a higher effect size, this did not deeply affect the result in the haemolysate (see online suppl. data S11).

Only 2 follow-up ischaemic stroke studies that investigated the GPx activity in plasma were found, reporting an increase after 7 days from ischaemic stroke onset compared to the value in the same patients at admission (SMD: 0.64, 95\% CI: 0.35-0.93, Fig. 5b). Two studies showing, respectively, the follow-up from ischemic and haemorrhagic stroke patients that evaluated serum were identified. The GPx activity was not significantly different in ischaemic stroke patients after 21 days from stroke onset compared to the same patients at admission (SMD: $0.09,95 \%$ CI: -0.32 to 0.51 , Fig. 5 c). In contrast, the only study analyzing the GPx activity in haemorrhagic stroke patients' serum showed a significant increase of the GPx activity in haemorrhagic stroke patients after 10-11 days from stroke onset compared to the same patients at admission (SMD: 9.46, 95\% CI: 6.28-12.69, online suppl. Fig. S12).

\section{GPx Activity Compared to Stroke Severity and Outcome}

For ischaemic stroke studies, an additional analysis was performed through comparison between measurements of GPx activity in patients grouped by the NIHSS and mRS outcome. Only 2 studies sub-divided the stroke patients by the NIHSS. From these, in both haemolysate and plasma compartments, the GPx activity in patients with a higher stroke severity (NIHSS > 10) was increased compared to mild or moderate stroke severity patients (NIHSS $\leq 10$; SMD: 0.67, 95\% CI: 0.25-1.10; MD: 4.57 U/g protein, 95\% CI: 3.96-5.18; online suppl. Fig. S13).

Furthermore, 2 studies analyzed the GPx activity in the haemolysate through sub-grouping the stroke patients into severe $(\mathrm{mRS}>3)$ and mild $(\mathrm{mRS} \leq 3)$ outcomes. A meta-analysis from these 2 studies showed that a decrease in the GPx activity in the haemolysate may be related to worse outcome in ischaemic stroke patients (MD: $-4.62 \mathrm{U} / \mathrm{gHb}, 95 \% \mathrm{CI}:-7.77$ to -1.47 , online suppl. Fig. S14).

\section{Discussion}

In this systematic review, we aimed to investigate whether selenoprotein concentrations or activity changes in acute ischaemic and haemorrhagic stroke patients. Despite the use of 17 different entry terms to identify all the records containing selenoprotein measurement, only studies showing GPx change after stroke were identified. Overall, we found that the GPx activity was not consistent between the 2 types of stroke. However, since only a few studies were identified investigating the role of GPx after haemorrhagic stroke, a comparison between GPx activity in ischaemic and haemorrhagic stroke patients was not possible.

In haemolysate, from both ischaemic and haemorrhagic stroke patients assessed within 3 days of stroke onset and compared to non-stroke controls, the hetero- 
geneity of the activity was $>90 \%$. This high heterogeneity, mostly due to contradictory results between the studies, may be explained by significant differences of patient and control characteristics in terms of confounding factors. In ischaemic stroke patients, the haemolysate GPx activity was found to decrease, increase, or stay unchanged compared to non-stroke controls. Of those studies, 2 recruited first-ever stroke patients and only one provided information about previous history of stroke (online suppl. Table S4). Likewise, conflicting results of haemolysate GPx activity are shown in haemorrhagic stroke patients compared to non-stroke controls. Although most of the patients' medical history or their lifestyle information was not reported, it is possible that previous stroke history may in part condition the amount of GPx in erythrocytes in further strokes, and this could potentially explain the large heterogeneity in reported findings. Due to the absence of long-term follow-up studies or studies showing patients sub-grouped by their previous stroke history, the latter hypothesis cannot be assessed in this review.

In contrast to haemolysate, in plasma, serum, and whole blood of ischaemic stroke patients assessed within 3 days of stroke onset, GPx activity and concentration decreased compared to non-stroke controls. Although only 7 studies investigated this aspect, the consistency of their results and the absence of heterogeneity suggest a high reliability. However, these results are not comparable to what the excluded study [31] reported, which was a significant increase of GPx activity in ischaemic stroke patients within 7 days of stroke onset compared to nonstroke controls. The reduction of GPx activity in plasma, serum, and whole blood may be caused by a decrease in the concentration of GPx3 isoform in particular, which is the major antioxidant enzyme in plasma. Accordingly, Akhter et al. [32] showed a significant decrease in GPx3 concentration in ischaemic stroke patients during the early acute phase.

Compared to patients with ischaemic stroke, in both blood compartments, the GPx activity from ICH and subarachnoid haemorrhage patients was unchanged compared to non-stroke controls. However, the only 2 identified studies, which included $<20$ patients each, are in conflict with a recently published study (not included in this review because it was published after the search was performed), showing that in patients with subarachnoid haemorrhagic stroke, there was a $133 \%$ increase of the plasma GPx activity compared to non-stroke controls [33].
The follow-up meta-analyses included 8 studies. Most of them $(n=7)$ concerned the follow-up of ischaemic stroke patients, whereas only 1 investigated the follow-up of haemorrhagic stroke patients. In the haemolysate of ischaemic stroke patients, the GPx activity remains elevated 7 days after stroke onset. However, the heterogeneity of the activity in this analysis was high. This can be in part attributable to the anomalous result from 1 study which used a different approach to prepare the haemolysate and biochemically assess GPx activity. However, this would not explain such high inconsistency of results of this study compared to the others.

In plasma and serum of follow-up ischaemic stroke patients, the GPx activity reached the non-stroke control values, and these results are in line with what Akhter et al. [32] reported on GPx3. Regarding haemorrhagic stroke, only 1 follow-up study on subarachnoid haemorrhage was found. This showed an approximately 70 fold increase of the GPx activity in serum of follow-up patients compared to the same patients at admission and to nonstroke controls. However, considering the low sample size from this study and a more recent opposing result from Jarocka-Karpowicz et al. [33], showing a decrease of plasma GPx activity in the follow-up of subarachnoid haemorrhagic stroke patients compared to the same patients within $24 \mathrm{~h}$ of admission, further investigation should be conducted.

Analyses of stroke severity suggest that an increase in haemolysate and plasma GPx activity might correlate with higher NIHSS. However, these 2 meta-analyses either resulted in high heterogeneity or included only 1 study. Furthermore, these results differ from the excluded study by Alexandrova et al. [31], where GPx activity in whole blood did not reflect the severity of neurological deficit measured with the Mathew scale.

Regarding haemorrhagic stroke, no studies were found comparing GPx activity or concentration in the blood in relation to the neurological scale. However, a recent study from Jarocka-Karpowicz et al. [33], assessed plasma GPX concentration relating to the World Federation of Neurological Surgeons Scale (WFNS). Although no differences were found in patients with worse neurological condition, this study showed a non-significant trend in increase in plasma GPx concentration in patients with brain edema compared to those with no edema [33].

Finally, a meta-analysis comparing the GPx activity of ischaemic stroke patients and mRS outcome was performed. The only 2 studies found showed a significant decrease of the haemolysate GPx activity in patients with worse outcome. A decrease in the GPx activity of eryth- 
rocytes may cause lipid peroxidation of their cellular membranes with consequent erythrocyte haemolysis [15], which may result in a worse outcome after stroke. However, further analyses are necessary to confirm these data.

Study quality of the articles was assessed using a modified 14-point NIHLB questionnaire, finding a mean score of 6 and generally fair quality. Yet, poor information of the eligibility rate, justification of sample size, blind assessment, and confounding variables were identified. Furthermore, the small number of studies and their small sample size did not allow for the creation of a reliable funnel plot to assess publication bias, and, since 7 studies were excluded because they were written in a different language (Russian and Spanish) other than English and Italian, this may have led to some bias. Sub-group analyses (see online suppl. data S7, S8, S11) by study quality (poor vs. fair/good) suggested a relationship between study quality and effect size, with the poor quality studies tending to report more extreme effect sizes.

\section{Limitations}

This systematic review has some limitations, which are mainly related to the characteristics of the studies included. Despite efforts to minimize the variance between the included studies through standardization of the units of measurement, some of them showed large differences in terms of result direction and magnitude compared to the other studies. This might in part be due to technical variation in the methodology to assess the GPx activity. Heterogeneity may also be to do with bias introduced by the design of these observational studies; 4 of the studies were rated as "poor quality" using the modified 14-point NIHLB questionnaire. Sub-group analyses revealed that effect size might be related to study quality.

The high variance found, especially in the haemolysate compartment, make the results from these analyses difficult to draw conclusions from. Another important limitation is represented by the restriction of this systematic review to the blood compartment. Selenoprotein changes in the central nervous system, where oxidative stress-induced damage is more considerable, were not addressed. To the best of our knowledge, there is only 1 study addressing selenoprotein measurement in autopsied brains of patients with or without cerebral infarction. Tazikawa et al. [34] described the cellular origin of GPx in the brain, showing that, whereas in the control tissue GPx staining was limited to neurons and glial cells, in ischaemic stroke patients, GPx staining was also found in the cytosol of macrophages within the core lesion.
However, no studies were found investigating GPx concentration or activity in the brain. In contrast, several studies with contradictory results analyzed how GPx activity changed in the cerebrospinal fluid (CSF) of haemorrhagic stroke patients. Some of them showed no significant differences between patients with ruptured or unruptured aneurism [35] and patients with or without ICH [36]. However, Pyne-Geithman et al. showed a significant increase of the GPx activity in the CSF of subarachnoid haemorrhagic stroke patients, which was raised in the presence of vasospasm [37]. Accordingly, Papacocea et al. [38] also found significantly increased GPx in the CSF of ICH stroke patients compared to nonstroke controls. It is evident that the behaviour of GPx may be specific to the stroke type. However, further studies are required to better understand the role of GPx in the pathophysiology of stroke.

\section{Conclusion}

This systematic review shows that there is a distinct lack of evidence to define all the selenoprotein trends after stroke. However, GPx analysis suggests a reduction of GPx activity in plasma, serum, and whole blood of ischaemic stroke patients assessed within 3 days of stroke onset compared to non-stroke controls. This may be due to a decrease in GPx3 concentration in these compartments, with this being the major isoform transported in the blood. In addition, this review suggests that a decrease in the erythrocyte GPx activity might correlate with worse outcome in ischaemic stroke patients. The inconsistency of the further results from this review and the small number of studies meeting the inclusion criteria make it impossible to draw any other conclusion. Therefore, further high-quality studies are required to provide more insights on how selenoprotein concentration and activity change after stroke and to identify whether selenium supplementation may induce a favourable effect on patients with stroke. Future observational studies should (i) consider higher sample sizes for adequate statistical power; (ii) report more fully details about patients' medical history and consider possible confounding factors, such as dietary vitamin and mineral intake of patients and controls; (iii) use a clearly stated reproducible and universal method to measure selenoprotein concentration and activity; and (iv) use a longitudinal cohort study, where the stroke population is followed over time. 


\section{Statement of Ethics}

An ethics statement was not required for this study type; no human or animal subjects or materials were used.

\section{Conflict of Interest Statement}

The authors declare no conflicts of interest.

\section{Funding Sources}

This work was supported by the Medical Research Council (MRC) Doctoral Training Partnership (DTP).

\section{Author Contributions}

S.T. and T.N.S. conceived the study and S.T. designed it. S.T., I.-E.M., L.R., and M.E.D.H. performed the systematic review, with input from S.M.A., C.J.S., T.N.S., and S.R. S.T. performed the statistical analysis assisted by S.R. S.M.A., C.J.S., T.N.S., S.R., and J.R.G. provided input to the study design, data interpretation, and commented on draft manuscript.

\section{Data Availability Statement}

All data generated or analyzed during this study are included in this article and its online supplementary material files. Further enquiries can be directed to the corresponding author.

\section{References}

1 Shirley R, Ord EN, Work LM. Oxidative stress and the use of antioxidants in stroke. Antioxidants. 2014;3(3):472-501.

2 Imai H, Graham DI, Masayasu H, Macrae IM. Antioxidant ebselen reduces oxidative damage in focal cerebral ischemia. Free Radic Biol Med. 2003;34(1):56-63.

3 Alim I, Caulfield JT, Chen Y, Swarup V, Geschwind DH, Ivanova E, et al. Selenium drives a transcriptional adaptive program to block ferroptosis and treat stroke. Cell. 2019;177(5): 1262-79.e25.

4 Ahmad A, Khan MM, Ishrat T, Khan MB, Khuwaja G, Raza SS, et al. Synergistic effect of selenium and melatonin on neuroprotection in cerebral ischemia in rats. Biol Trace Elem Res. 2011;139(1):81-96.

5 Takasago T, Peters EE, Graham DI, Masayasu $\mathrm{H}$, Macrae IM. Neuroprotective efficacy of ebselen, an anti-oxidant with anti-inflammatory actions, in a rodent model of permanent middle cerebral artery occlusion. Br J Pharmacol. 1997;122(6):1251-6.

6 Brigelius-Flohé R, Maiorino M. Glutathione peroxidases. Biochim Biophys Acta Gen Subj. 2013;1830(5):3289-303.

7 Di Marzo N, Chisci E, Giovannoni R. The role of hydrogen peroxide in redox-dependent signaling: homeostatic and pathological responses in mammalian cells. Cells. 2018; 7(10): 156

8 Grond-Ginsbach C, Lichy C, Padovani A, Pezzini A. GPx-3 gene promoter variation and the risk of arterial ischemic stroke. Stroke. 2007;38(6):e23; author reply e24

9 Voetsch B, Jin RC, Bierl C, Deus-Silva L, Camargo EC, Annichino-Bizacchi JM, et al. Role of promoter polymorphisms in the plasma glutathione peroxidase $(\mathrm{GPx}-3)$ gene as a risk factor for cerebral venous thrombosis. Stroke. 2008;39(2):303-7.

10 Voetsch B, Jin RC, Bierl C, Benke KS, Kenet $\mathrm{G}$, Simioni $\mathrm{P}$, et al. Promoter polymorphisms in the plasma glutathione peroxidase (GPx-3) gene: a novel risk factor for arterial ischemic stroke among young adults and children. Stroke. 2007;38(1):41-9.

11 Yamaguchi T, Sano K, Takakura K, Saito I, Shinohara Y, Asano T, et al. Ebselen in acute ischemic stroke: a placebo-controlled, double-blind clinical trial. Ebselen Study Group. Stroke. 1998;29(1):12-7.

12 Demirkaya S, Topcuoglu MA, Aydin A, Ulas UH, Isimer AI, Vural O. Malondialdehyde, glutathione peroxidase and superoxide dismutase in peripheral blood erythrocytes of patients with acute cerebral ischemia. Eur J Neurol. 2001;8(1):43-51.

13 Ljubisavljevic S, Cvetkovic T, Zvezdanovic L, Stojanovic S, Stojanovic I, Kocic G, et al. The differences in the cellular and plasma antioxidative capacity between transient and defined focal brain ischemia: does it suggest supporting time-dependent neuroprotection therapy? Cell Mol Neurobiol. 2016;36(5): 789-800.

14 Ozbey U, Seyran A, Erisir M, Ozel S. Oxidative stress and altered levels of oxidants and antioxidants in acute ischemic stroke patients in a region of east turkey. HealthMED. 2012; 6(5):1574-82.

15 Sudha K, Rao AV, Rao S, Rao A. Lipid peroxidation, hemolysis and antioxidant enzymes of erythrocytes in stroke. Indian J Physiol Pharmacol. 2004;48(2):199-205.

16 Žitňanová I, Šiarnik P, Kollár B, Chomová M, Pazderová P, Andrezálová L, et al. Oxidative stress markers and their dynamic changes in patients after acute ischemic stroke. Oxid Med Cell Longev. 2016;2016:9761697.

17 Kolesnichenko LS, Kulinski VI, Shprakh VV, Bardymov VV, Verlan NV, Gubina LP, et al. [Glutathione system in erythrocytes and blood plasma in strokes and dyscirculatory encephalopathy]. Biomed Khim. 2007;53(4): 454-60.

18 Chen YC, Chen CM, Liu JL, Chen ST, Cheng ML, Chiu DT. Oxidative markers in sponta- neous intracerebral hemorrhage: leukocyte 8-hydroxy-2' -deoxyguanosine as an independent predictor of the 30-day outcome. J Neurosurg. 2011;115(6):1184-90.

19 Lutsky MA, Zemskov AM, Razuvaeva VV, Lushnikova YP, Karpova OY. [Oxidative stress as an indicator of metabolic disorders in the pathogenesis of cerebral stroke]. Zh Nevrol Psikhiatr Im S S Korsakova. 2017;116(8 Pt 2):24-9.

20 Aygul R, Kotan D, Demirbas F, Ulvi H, Deniz O. Plasma oxidants and antioxidants in acute ischaemic stroke. J Int Med Res. 2006;34(4): 413-8.

21 Cherubini A, Polidori MC, Bregnocchi M, Pezzuto $S$, Cecchetti R, Ingegni T, et al. Antioxidant profile and early outcome in stroke patients. Stroke. 2000;31(10):2295-300.

22 Cojocaru IM, Cojocaru M, Sapira V, Ionescu A. Evaluation of oxidative stress in patients with acute ischemic stroke. Rom J Intern Med. 2013;51(2):97-106

23 Aygul R, Demircan B, Erdem F, Ulvi H, Yildirim A, Demirbas F. Plasma values of oxidants and antioxidants in acute brain hemorrhage: role of free radicals in the development of brain injury. Biol Trace Elem Res. 2005; 108(1-3):43-52.

24 Liu Z, Zhu Z, Zhao J, Ren W, Cai Y, Wang Q, et al. Malondialdehyde: a novel predictive biomarker for post-stroke depression. J Affect Disord. 2017;220:95-101.

25 Milanlioglu A, Aslan M, Ozkol H, Çilingir V, Nuri Aydın M, Karadas S. Serum antioxidant enzymes activities and oxidative stress levels in patients with acute ischemic stroke: influence on neurological status and outcome. Wien Klin Wochenschr. 2016;128(5-6):16974.

26 Yildirim A, Kotan D, Yildirim S, Aygul R, Akcay F. Increased lipid peroxidation and decreased antioxidant response in serum and cerebrospinal fluid in acute ischemic stroke. Turk J Med Sci. 2007;37(2):75-81. 
27 Zimmermann C, Winnefeld K, Streck S, Roskos M, Haberl RL. Antioxidant status in acute stroke patients and patients at stroke risk. Eur Neurol. 2004;51(3):157-61.

28 Watanabe T, Sasaki T, Asano T, Takakura K, Sano K, Fuchinoue T, et al. Changes in glutathione peroxidase and lipid peroxides in cerebrospinal fluid and serum after subarachnoid hemorrhage: with special reference to the occurrence of cerebral vasospasm. Neurol Med Chir. 1988;28(7):645-9.

29 Sheikh N, Tavilani H, Rezaie A, Vaisi-raygani A, Salimi S. Relationship between estradiol and antioxidant enzymes activity of ischemic stroke. J Biomed Biotechnol. 2009;2009: 841468.

30 Wan X, Wang W, Liu J, Tong T. Estimating the sample mean and standard deviation from the sample size, median, range and/or interquartile range. BMC Med Res Methodol. 2014;14:135.
31 Alexandrova M, Bochev P, Markova V, Bechev B, Popova M, Danovska M, et al. Dynamics of free radical processes in acute ischemic stroke: influence on neurological status and outcome. J Clin Neurosci. 2004;11(5):501-6.

32 Akhter MS, Biswas A, Rashid H, Devi L, Behari M, Saxena R. Screening of the GPX3 gene identifies the "T" allele of the SNP -861A/T as a risk for ischemic stroke in young Asian Indians. J Stroke Cerebrovasc Dis. 2014;23(8): 2060-8.

33 Jarocka-Karpowicz I, Syta-Krzyżanowska A, Kochanowicz J, Mariak ZD. Clinical prognosis for SAH consistent with redox imbalance and lipid peroxidation. Molecules. 2020; 25(8): 1921.

34 Takizawa S, Matsushima K, Shinohara Y, Ogawa S, Komatsu N, Utsunomiya $\mathrm{H}$, et al. Immunohistochemical localization of glutathione peroxidase in infarcted human brain. J Neurol Sci. 1994;122(1):66-73.
35 Gaetani P, Pasqualin A, Rodriguez y Baena R, Borasio E, Marzatico F. Oxidative stress in the human brain after subarachnoid hemorrhage. J Neurosurg. 1998;89(5):748-54.

36 Mantle D, Siddique S, Eddeb F, Mendelow AD. Comparison of protein carbonyl and antioxidant levels in brain tissue from intracerebral haemorrhage and control cases. Clin Chim Acta. 2001;312(1-2):185-90.

37 Pyne-Geithman GJ, Caudell DN, Prakash P, Clark JF. Glutathione peroxidase and subarachnoid hemorrhage: implications for the role of oxidative stress in cerebral vasospasm. Neurol Res. 2009;31(2):195-9.

38 Papacocea T, Buraga I, Papacocea R, Badarau IA, Buraga M, Ciornei C, et al. Antioxidant enzymes-potential targets in intracerebral haemorrhage. Farmacia. 2014;62(6):1118-25. 